\title{
S.A.Mustafayeva, V.A.Mirzazada
}

Azerbaijan State Institute for Postgraduate Education of Doctors (Baku, Azerbaijan)

Background. The threat and incidence of hypoglycemia is the major limiting factor in intensive glycemic control for all types of diabetes mellitus (DM).

The aim of this work was to study frequency and depth of hypoglycemia episodes at patients with diabetes type 2 (DM2) in Azerbaijan by using Continuous Glucose Monitoring (CGM).

Methods. 50 out-patients (30 women and 20 men) with DM2 were studied. (table 1)

Two CGM devices have been used in this research: CGMS (Mini Med, USA) and Dexcom (Dexcom, USA). According to received treatment patients were divided in 2 groups: Group $1(n=34)$ - insulin therapy (mono- and in combination with tablets); Group $2(n=16)$ - peroral glucose-lowering therapy (mono-, dual and triple therapy). There were no statistically significant differences between two groups in age, duration of DM2, BMI, blood pressure and metabolic parameters, including $\mathrm{HbA} 1 \mathrm{c}$.

\begin{tabular}{|l|l|l|}
\hline & Group1 & Group2 \\
\hline Age(yrs) & $56,2 \pm 11,37$ & $51,5 \pm 12,07$ \\
\hline BMI(kg/m²) & $33,8 \pm 6,39$ & $30,8 \pm 4,74$ \\
\hline Duration of disease(yrs) & $14,7 \pm 7,91$ & $7,1 \pm 3,55$ \\
\hline Sistolic BP(mmHg) & $139,8 \pm 19,21$ & $136,6 \pm 18,5$ \\
\hline Diastolic BP(mmHg) & $86,3 \pm 9,48$ & $83,4 \pm 11,21$ \\
\hline HbA1c(\%) & $9,3 \pm 1,9$ & $8,0 \pm 1,43$ \\
\hline Triglycerides(mq/dl) & $195,8 \pm 59,04$ & $200 \pm 57,28$ \\
\hline HDL(mq/dl) & $47,3 \pm 5,68$ & $48,1 \pm 4,57$ \\
\hline LDL(mq/dl) & $107,9 \pm 32,42$ & $103,5 \pm 36,06$ \\
\hline Cholesterol(mq/dl) & $195,6 \pm 37,07$ & $191,2 \pm 43,41$ \\
\hline
\end{tabular}

Table 1
Results. The frequency of hypoglycaemia episodes at patients with DM2 was $42.9 \%$ in Group 1 vs. $37.5 \%$ in Group2 ( $p>0.05$ ). (Table 2). Total number of glucose determinations were 41,543 in Group 1 and 20,148 in Group 2. Number of measurements with hypoglycaemic results were 772 in Group 1 and 122 in Group 2. Average hypoglycemic glucose levels (glucose values of $69 \mathrm{mg} / \mathrm{dl}$ and below) were $53.9 \pm 10.89$ for Group 1 vs. $60.6 \pm 4.70$ for Group 2. Difference between two groups was statistically significant $(p<0.001)$.

Conclusion. Despite the high HbA1c levels in both groups hypoglycemia frequently complicated treatment of diabetes. The type of treatment had impact on decrease in glucose level. Lower levels of glucose were associated with insulin therapy.

Thus, our data have shown that the frequency of hypoglycemia is not different at cases of insulin therapy and peroral glucoselowering therapy, but hypoglycemic episodes at insulin therapy are more dangerous as they are associated with more essential decrease of glucose level.

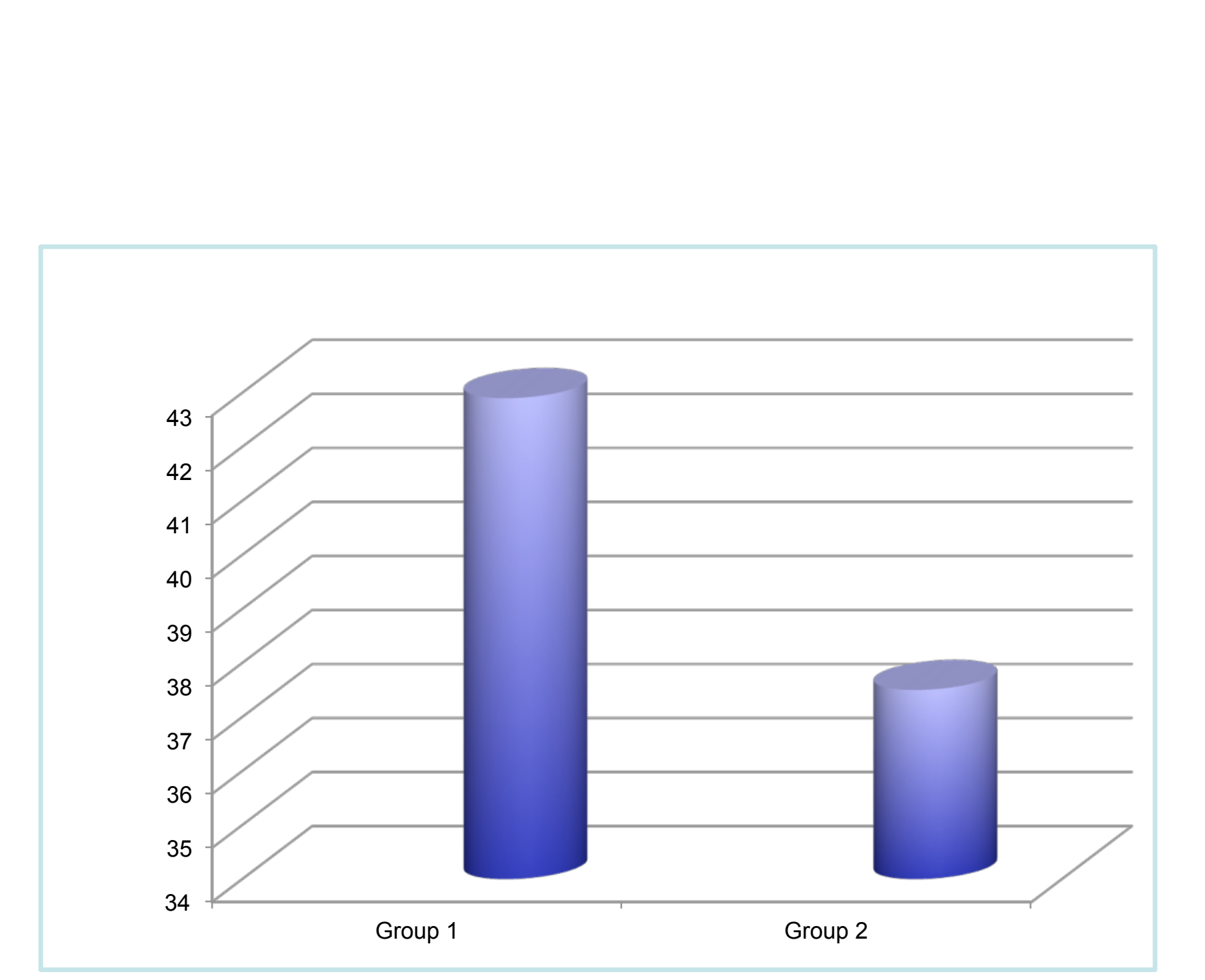

Table 2 